\title{
1 IDEA: a web server for Interactive Differential 2 Expression Analysis with R Packages
}

3 Qi Zhao ${ }^{1, \dagger}$, Yubin $\mathrm{Xie}^{1, \dagger}$, Peng Nie ${ }^{1, \dagger}$, Rucheng Diao ${ }^{1}$, Lichen Sun ${ }^{1}$, Zhixiang Zuo ${ }^{1, *}$, $4 \quad$ Jian $\operatorname{Ren}^{1, *}$

$6 \quad{ }^{1}$ State Key Laboratory of Oncology in South China, Cancer Center, Collaborative Innovation

7 Center for Cancer Medicine, School of Life Sciences, Sun Yat-sen University, China

$8 \quad$ *To whom correspondence should be addressed.

9 TThe authors wish it to be known that, in their opinion, those authors contributed equally to this work.

\section{Abstract}

12 Differential expression (DE) analysis is a fundamental task in the downstream analysis of the 13 next-generation sequencing (NGS) data. Up to now, a number of $\mathrm{R}$ packages have been 14 developed for detecting differentially expressed genes. Although $\mathrm{R}$ language has an 15 interaction-oriented programming design, for many biology researchers, a lack of basic 16 programming skills has greatly hindered the application of these $\mathrm{R}$ packages. To address this 17 issue, we developed the Interactive Differential Expression Analyzer (IDEA), a Shiny-based 18 web application integrating the differential expression analysis related $\mathrm{R}$ packages into a 19 graphical user interface (GUI), allowing users to run the analysis without writing any new code.

20 A wide variety of charts and tables are generated to facilitate the interpretation of the results.

21 In addition, IDEA also provides a combined analysis framework which helps to reconcile any 22 discrepancy from different computational methods. As a public data analysis server, IDAE is 23 implemented in HTML, CSS and JavaScript, and is freely available at http://idea.renlab.org.

25 Keywords: differential expression analysis, interactively analysis, integrated analysis. 


\section{Introduction}

28

29 High-throughput sequencing technology is rapidly becoming the standard method for

30 measuring gene expression at the transcriptional level. One of the major goals of such work is

31 to identify differentially expressed (DE) genes under two or more conditions. A number of 32 computational tools, such as DESeq2 [1], edgeR [2], NOISeq [3], PoissonSeq [4], SAMseq [5]

33 and Cuffdiff [6] have been developed for the analysis of differential gene expression from 34 RNA-seq data. Most of these tools are implemented in R language, which is commonly used 35 for the analysis of high-dimensional expression data. However, a fairly high level of 36 programing skill is required when applying these $\mathrm{R}$ tools to screen out differentially expressed 37 genes, which greatly hinders the application of these tools since many biology researchers have little programing experience. In recent year, a number of software, such as easyRNASeq[7], RNASeqGUI[8] and RobiNA[9] et al, was developed to address this issue. Although the above tools have provided convenient GUI-based computer platforms for back-end $\mathrm{R}$ packages to some extent, none of them implements an interactive interface that can not only facilitate the understanding of analysis process for a web-bench scientist, but also inconvenient to adjust the analytical parameters, even for advanced users. Moreover, since different packages will generate inconsistent results, it is difficult for the users to decide which

45 DE algorithm to use. Thus, an interactive platform that can combine these tools together is necessary for obtaining more solid analysis results.

47 In this regards, we present the Interactive Differential Expression Analyzer (IDEA), a

48 Shiny-based web application dedicated to the identification of differential expression genes in 49 an interactive way. IDEA was built as a user-friendly and highly interactive utility using the 50 Shiny package in $R$. Besides, five relevant $R$ packages are integrated into IDEA. IDEA is 51 capable of visualizing the results with plenty of charts and tables, as well as providing great 52 ease of interaction during the analysis 


\section{Features and options}

56 Framework

57 IDEA provides a well-designed data analysis framework to help identify reliable differential 58 expressed features (Figure 1, 2A). Briefly, three kinds of experimental comparison between 59 groups can be performed using IDEA, namely "standard comparison", "multifactor design" and 60 "comparison without replicates". To have a quick start, IDEA requires users to specify the 61 experimental design and can automatically determine available packages for further analysis.

62 For input dataset, a read count matrix and a design matrix which presents the relationship 63 between samples and conditions were mandatory for an independent analysis. Users were 64 encouraged to load feature length file to obtain length-normalized expression values, such as 65 (Reads Per Kilobase Million) RPKM and (Fragments Per Kilobase Million) FPKM. After that, data exploration panel was introduced to help assessing the data quality and normalizing the

67 reads count dataset for further analysis. Differential expression analysis can be sequentially 68 selected for running separately or not. As different algorithms may generate inconsistent 69 differential expression feature list, we further developed a combine analysis module in IDEA to 70 integrate result from each selected analysis packages. Additionally, a build-in example data 71 that enabled users to run demo analysis and download demo report was established.

\section{Data exploration}

74 Data quality assessment and quality control are essential steps for expression analysis.

75 Beyond the reads base quality and mapping quality evaluation in raw data preprocessing 76 stage, IDEA implements a quality control panel based on reads count distribution in terms of 77 genes and samples. In this panel, we provide several normalized methods including TPM, 78 upper quartile (Default), RPKM and "none" to help exploring potential calculating bias from 79 different normalization approaches. We also provide three visualization strategies: density 80 area plot, bar plot and box plot to help assessing the data quality (Figure 2B). These plots 81 illustrate the feature expression distribution in each sample, allowing users to identify samples with extremely low or high expressed gene number. 
83 Expression-based principal component analysis (PCA) and correlation analysis were provided

84 for studying the sample relationship. Scatter plot with sample point and heat map presenting

85 spearman correlation coefficients were generated respectively. Specifically, if the samples

86 come from the same condition, we expect to observe a closer sample distance in the scatter

87 plot, and to find the tested samples under the resembling branch within clustered heat map.

88 Generally, biological or technical replicates exhibit higher correlation than samples from

89 different sources, thus an extra correlation scatter plot containing detail relationships between

90 couple of samples was included. It gives a further investigation of correlation analysis result

91 from clustered heat map.

92 It's also crucial for biologist to verify the known or some housekeeping genes in the experiment.

93 By setting negative and positive control in a "wet" experiment, features with known expression

94 patterns can be applied for assessing the quality of sequencing library, such as low expression

95 of a gene in knockout library against wild type. IDEA, therefore, provides a bar plot interface to

96 help users query the average abundance of a specified gene in each condition, while some

97 internal control features can be checked with the help of this function.

98

99 Multiple DE methods integration

100 To allow users with little programming experience to easily perform DE analysis using the

101 most commonly used R packages, the current implementation of IDEA has integrated DESeq2,

102 edgeR, NOISeq, PossionSeq and SAMseq into a convenient web-based GUI. To avoid

103 tedious operations, parameters of each method were optimized to fit most cases, and only few

104 ones were required be adjusted in special scenario. When the analysis is finished, important

105 information involved in each method was also presented, including model parameters

106 estimation, normalized factor and statistic descriptions. Plots, like MA plot, volcano plot (Figure

$1072 \mathrm{2C}$ ), clustered heat map and FDR/P-value distribution bar plot, are presented to summarize

108 the identified DE genes.

109 Different packages may generate discrepant results on the same dataset. To reconcile any

110 such discrepancy, we developed a combination analysis module to integrate the results from 
111 the different packages in an unbiased manner. Using a set of rank aggregation methods [10],

112 such as Robust Rank Aggregation and Stuart P-value Integration, the integration score of a

113 given DE feature is calculated as the mean of the integrated rank numbers generated by each

114 of the packages. To visualize the combined results, a bar plot showing the DE features

115 identified by each packages (Figure 2D) and a Venn diagram demonstrating the distribution

116 and reliability of the verified features (Figure 2E) were developed. Finally, the combined result

117 was also presented in an interactive table to help exploring the most confidential DE features

118 (Figure 2F).

119

120 Interactive analysis and fancy analysis report

121 Interactive features involved in IDEA consist of dynamic data operation, parameter adjustment

122 and real-time plot rendering. First, dynamic data table enables interactively filtering, sorting

123 and querying genes from input and out data frames by sorts of attributes (Figure 3A). Second,

124 parameter adjustment enables users to refine analysis with modified parameters in real time

125 without rerun the irrelevant step. For example, in DEseq analysis section, modifying DE gene

126 filtering criterion only require to trigger transformation of the result table, and the DEseq will not

127 be executed again. Similarly, in combine analysis, modifying the parameters of one specific

128 tool do not affect others. This may greatly reduce the reanalysis time for exploring analysis,

129 and especially suitable for screening gene lists that are stable under different parameters. For

130 interactive plot rendering, we added some switchable panels in plot frame for refining plot

131 setting in real time. Samples, colors, comparisons and plot data can be dynamically adjusted

132 as well (Figure 3B, 3C). Those options can just trigger re-execution of the plot function while

133 leave the computing function unchanged. Plots can be interactive repainted while the

134 corresponding parameters altered.

135 Using $\mathrm{R}$ markdown and knitr package, IDEA summarized analysis results into a

136 well-structured fancy report with all parameters, figures and citations listed. To meet the

137 publication requirement, high quality figures with $300 \mathrm{dpi}$ and result table can be downloaded

138 from the final report, separately. 


\section{Implementation}

141 IDEA was initially implemented in R programming language [https://www.r-project.org/] with 142 shiny [http://shiny.rstudio.com/] for building interactive web applications. It hosted in a Unix-like

143 system which have both R and shiny server pre-installed. The front-end code was elaborately 144 reconstructed with JavaScript, CSS and html to optimize the interactive experience of users.

145 We also implemented an R package version of IDEA that allows users to run analysis locally 146 under $\mathrm{R}$ environment. The package can be freely accessed and installed from 147 https://github.com/likelet/IDEA. Detailed usage information was presented at the 'help' section 148 of the web page.

\section{Future developments}

151 As IDEA was developed under shiny web framework, its web utilities were greatly limited by 152 shiny server performance. Therefore, to lift the limitation of server resources, we encourage 153 users to install the local version when performing differential expression analysis. Besides, for 154 interactive analysis, plots were dynamically rendered when data or parameters have changed, 155 while a true sense of interactive plot have not yet been implemented due to a possible heavy 156 browser loading. We will overcome this with reduced data points and customized data 157 structure in the near future. Popular data visualization JavaScript library like D3 158 (https://d3js.org), plotly (https://plot.ly) and Highchart (https://www.highcharts.com) will be 159 integrated as well. For the extension of more functions, extra analysis features, such as gene

160 annotation, function enrichment and co-expression analysis, will be included into IDEA in the 161 near future.

162 Nowadays, various third-party tools were developed to perform differential expression analysis

163 [11], while some of them were not implemented in R. In the current version, IDEA only 164 selected five most popular and efficient R-based tools in the analysis pipeline. For future 165 development, packages in low computing efficiency will be integrated in R package version as 166 an optional analysis module. However, non R-based tools like gfold[12], cufflinks[13] and 
167 Stringtie[14] etc, will not directly supported in our platform. Instead, to cope alternately with this

168 issue, we are going to provide a combine analysis framework based on results generated by

169 those tools.

170

171 Conclusion

172 IDEA is a user-friendly web application for differential expression analysis, which combines the

173 five commonly used DE analysis packages into a highly interactive web-based framework

174 using the Shiny package. The abundant visualization functions in IDEA enable the users to

175 gain both deeper insight into their original data and the ability to interpret the results in a more

176 intuitive way. Furthermore, in contrast to the static image generated by R language, IDEA can

177 produce diagrams in real time, which makes the analytical process more convenient and

178 efficient. Taken together, we introduce IDEA as an effective application that can facilitate and

179 simplify the process of differential expression analysis in high-dimension expression data.

180

181 Funding

182 This work was supported by grants from the National Natural Science Foundation of China

183 [31471252, 31771462, 81772614 and U1611261]; National Key Research and Development

184 Program [2017YFA0106700]; Guangdong Natural Science Foundation [2014TQ01R387 and

185 2017A030313134]; Science and Technology Program of Guangzhou, China [201604020003

186 and 201604046001]; and China Postdoctoral Science Foundation [2017M610573,

187 2017M622864 and 2018T110907].

188

189 References

190 1. Love MI, Huber W, Anders S. Moderated estimation of fold change and dispersion for

191 RNA-seq data with DESeq2, Genome Biol 2014;15:550.

192 2. Zhou X, Lindsay H, Robinson MD. Robustly detecting differential expression in RNA

193 sequencing data using observation weights, Nucleic Acids Res 2014;42:e91.

194 3. Tarazona S, Garcia-Alcalde F, Dopazo J et al. Differential expression in RNA-seq: a 
195 matter of depth, Genome Res 2011;21:2213-2223.

196 4. Li J, Witten DM, Johnstone IM et al. Normalization, testing, and false discovery rate

197 estimation for RNA-sequencing data, Biostatistics 2012;13:523-538.

198 5. Li J, Tibshirani R. Finding consistent patterns: a nonparametric approach for identifying

199 differential expression in RNA-Seq data, Stat Methods Med Res 2013;22:519-536.

200 6. Trapnell C, Hendrickson DG, Sauvageau M et al. Differential analysis of gene regulation

201 at transcript resolution with RNA-seq, Nat Biotechnol 2013;31:46-53.

202 7. Delhomme N, Padioleau I, Furlong EE et al. easyRNASeq: a bioconductor package for 203 processing RNA-Seq data, Bioinformatics 2012;28:2532-2533.

204 8. Russo F, Angelini C. RNASeqGUI: a GUI for analysing RNA-Seq data, Bioinformatics 205 2014;30:2514-2516.

206 9. Lohse M, Bolger AM, Nagel A et al. RobiNA: a user-friendly, integrated software solution 207 for RNA-Seq-based transcriptomics, Nucleic Acids Res 2012;40:W622-627.

208 10. Kolde R, Laur S, Adler $P$ et al. Robust rank aggregation for gene list integration and 209 meta-analysis, Bioinformatics 2012;28:573-580.

210 11. Conesa A, Madrigal $P$, Tarazona $S$ et al. A survey of best practices for RNA-seq data 211 analysis, Genome Biol 2016;17:13.

212 12. Feng J, Meyer CA, Wang $Q$ et al. GFOLD: a generalized fold change for ranking 213 differentially expressed genes from RNA-seq data, Bioinformatics 2012;28:2782-2788.

214 13. Trapnell C, Roberts $A$, Goff $L$ et al. Differential gene and transcript expression analysis of 215 RNA-seq experiments with TopHat and Cufflinks, Nat Protoc 2012;7:562-578.

216 14. Pertea M, Kim D, Pertea GM et al. Transcript-level expression analysis of RNA-seq 217 experiments with HISAT, StringTie and Ballgown, Nat Protoc 2016;11:1650-1667.

\section{Figure legends}

222 Figure 1. Differential expression analysis framework implemented in IDEA. 
223 Figure 2. Selected analysis plots generated by IDEA. A, screen shot of IDEA web page. B, a

224 box plot presents the normalized reads count distribution among samples. C, Differential

225 expression analysis result was shown in a volcano plot; each point represents a feature for

226 statistic test, the green points are significantly differential expressed with adjusted $\mathrm{P}$ value $<$

227 0.05. D, Overlap analysis based on the DE result from five analysis packages. E, overview of

228 differential expressed feature count by each package shown in a bar plot. F. combine analysis

229 result data frame, only top 50 features were displayed as default.

230 Figure 3. Interactive features provided in IDEA. A. dynamic data table JavaScript library was

231 applied to help interactively exploring features stored in data frame. B. The floating control

232 panel that enables modifying parameters in real-time.

233 


\section{$234 \quad$ Figure 1}

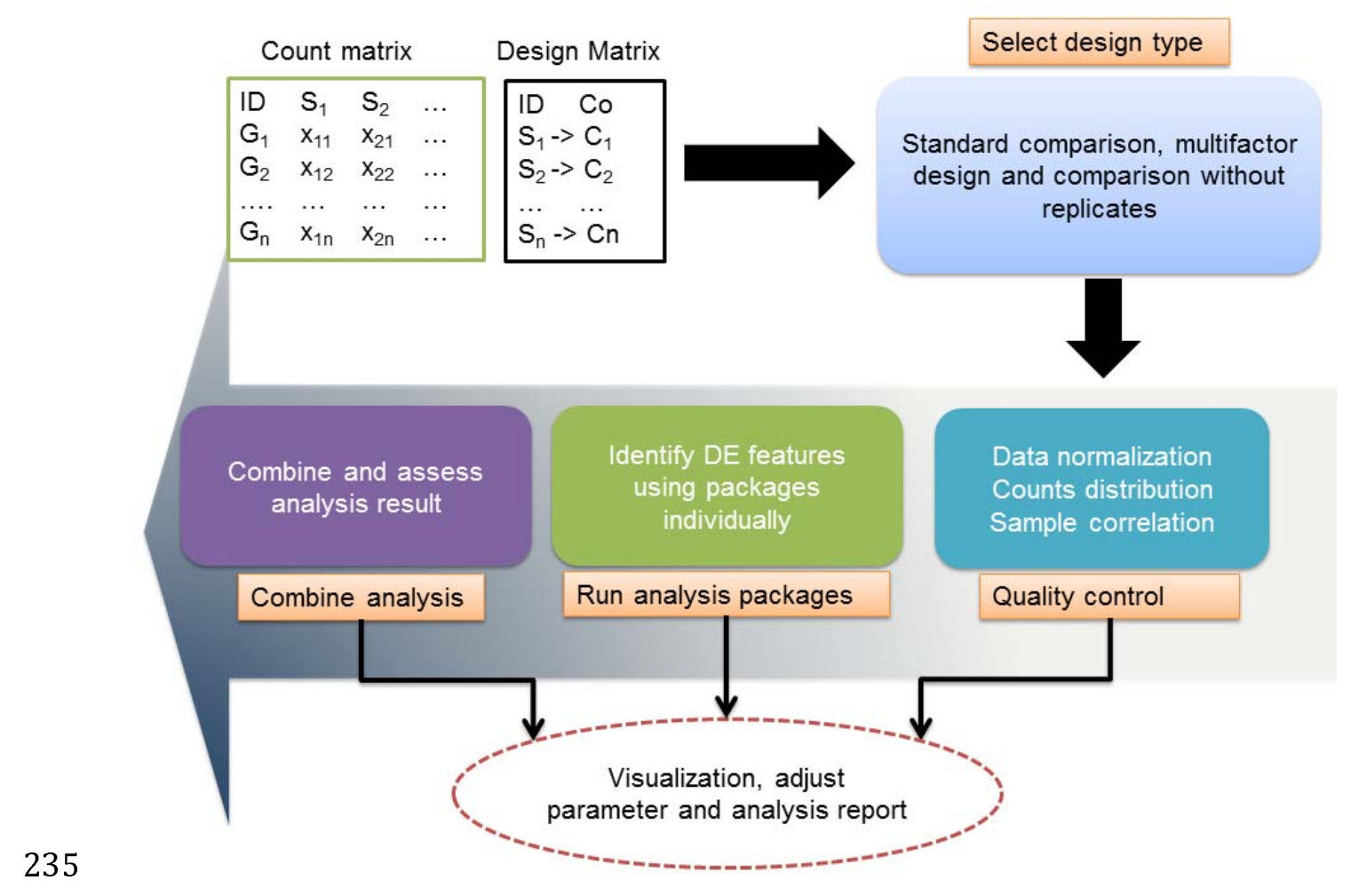


bioRxiv preprint doi: https://doi.org/10.1101/360461; this version posted July 3, 2018. The copyright holder for this preprint (which was not certified by peer review) is the author/funder. All rights reserved. No reuse allowed without permission.

\section{$237 \quad$ Figure 2}

A

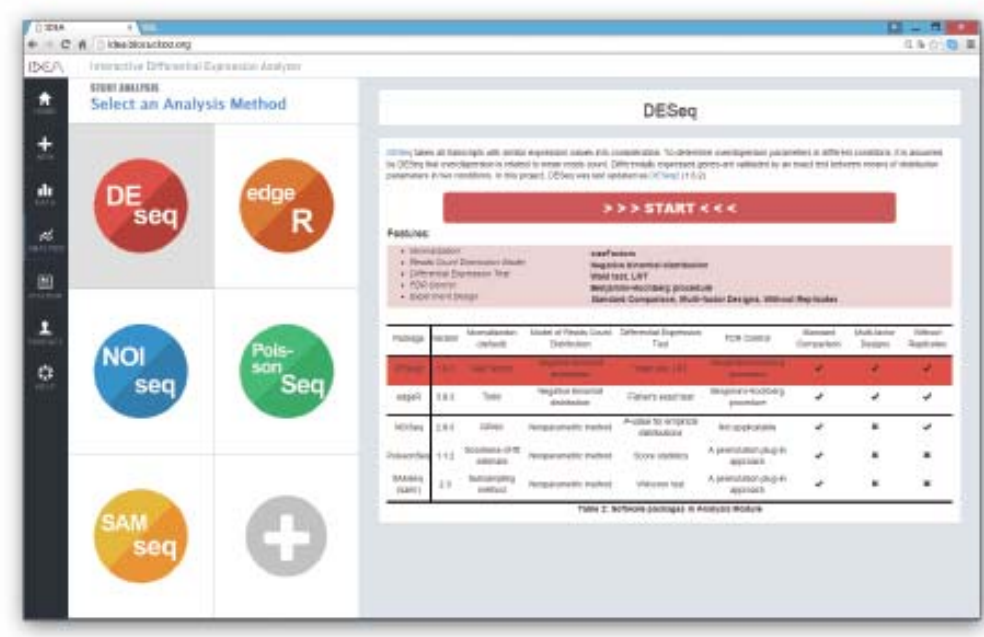

B

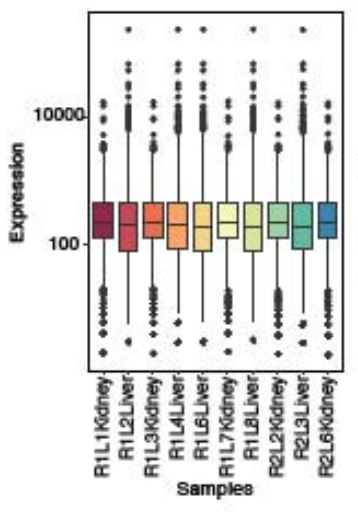

E

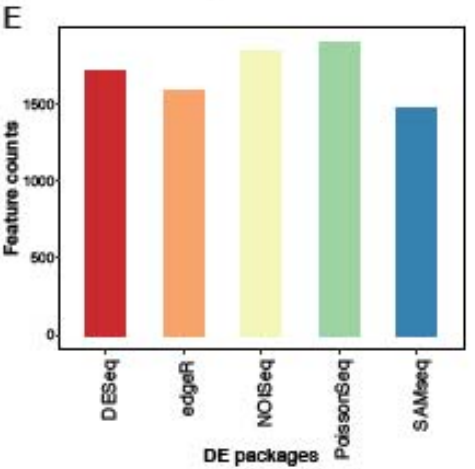

C

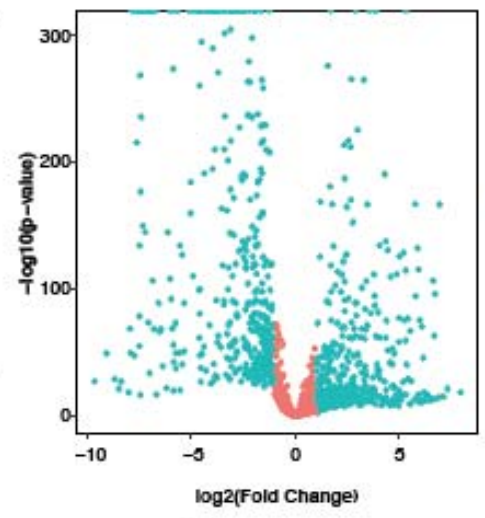

F

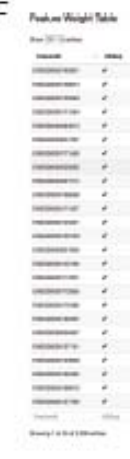

D
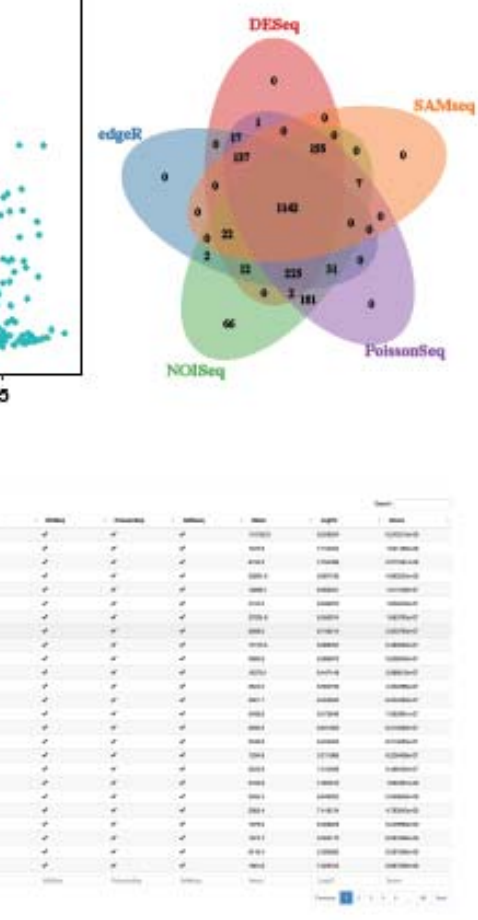
bioRxiv preprint doi: https://doi.org/10.1101/360461; this version posted July 3, 2018. The copyright holder for this preprint (which was not certified by peer review) is the author/funder. All rights reserved. No reuse allowed without permission.

\section{$240 \quad$ Figure 3}

241

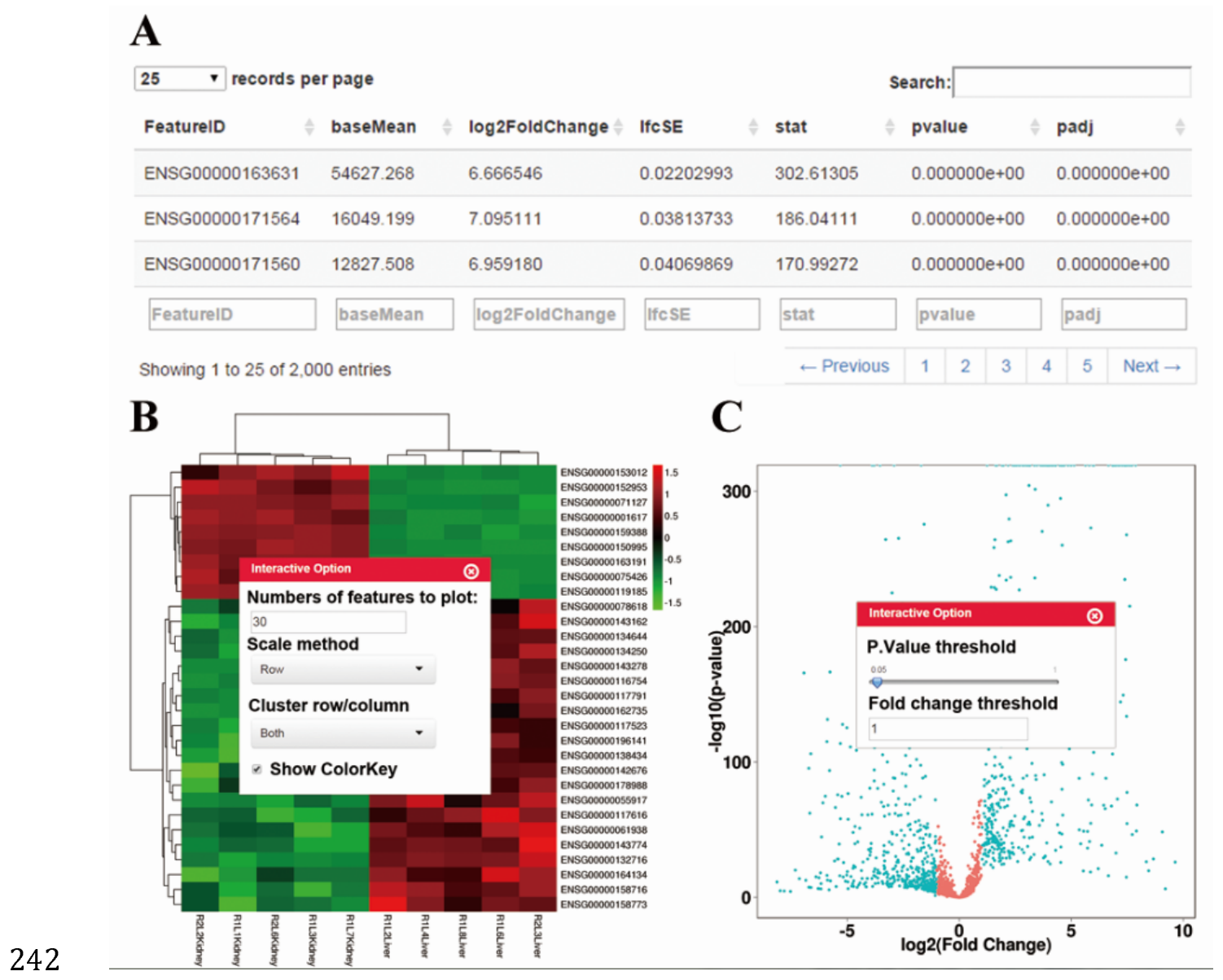

